\title{
PSEUDO-ORBITS AND TOPOLOGICAL ENTROPY
}

\author{
MARCY BARGE AND RICHARD SWANSON
}

(Communicated by Kenneth R. Meyer)

\begin{abstract}
The topological entropy of a map of a compact metric space is equal to the exponential growth rate of the number of separated periodic pseudo-orbits of period $n$ as $n$ tends to infinity.
\end{abstract}

\section{INTRODUCTION}

In the years since their development by Rufus Brown [B] and Charles Conley [C], pseudo-orbits have proved to be a powerful conceptual tool in dynamical systems. Grebogi, Hammel, and Yorke [HYG1, HYG2] have recently investigated the role of pseudo-orbits in computer simulations of certain dynamical systems; while Easton [E], McGehee [Mc], Hurley [H], and Franks [F] have exploited the rough dynamics and gradient-like behavior of pseudo-orbits. Also, in recent work, the authors [BS] have made use of pseudo-orbits to study rotation sets of circle and annulus maps. In this spirit, one can approach the study of topological entropy.

Topological entropy was first defined along with its basic properties in [AKM]. It is not too hard to verify that, for a subshift of finite type, the entropy is given by the exponential growth rate of the number of periodic orbits of period $n$ as $n$ tends to infinity. But for other subshifts or the pathological example in $[R]$ it is possible to have positive entropy without any periodic orbits whatsoever. What is the relation of periodic pseudo-orbits to entropy? We shall prove in Theorem 2 , our main result, that the topological entropy can be computed by measuring the exponential growth rate of the number of (separated) periodic pseudo-orbits of period $n$, as $n$ tends to infinity. This can be contrasted with the fact that, in order for positive entropy to force the existence of true periodic orbits, one must, in general, require both expansiveness and shadowing properties. However, at the coarse-grained level of pseudo-orbits, positive entropy is always accounted for by the exponential accumulation of large periodic orbits, which, perhaps, is in sympathy with our naive expectations.

Received by the editors July 23, 1989 and, in revised form, August 10, 1989.

1980 Mathematics Subject Classification (1985 Revision). Primary 58F11.

Both authors were supported by grants from MONTS-NSF. 
The authors would like to thank Mike Hurley for observing that, in 1984, Misiurewicz [Mi] considered the calculation of entropy from (not necessarily periodic) pseudo-orbits and proved Theorem 1 in the next section. We include a proof of that theorem for completeness.

\section{DEFINITIONS AND TERMINOLOGY}

Let $f: X \rightarrow X$ be a continuous map on the compact metric space $(X, d)$. An $\alpha$-pseudo-orbit is an infinite sequence $x_{0}, x_{1}, x_{2}, \ldots$ such that $d\left(f\left(x_{i}\right), x_{i+1}\right)$ $\leq \alpha$ for $i \geq 0$. If the sequence is finite, we will use the term $\alpha$-chain. The $\alpha$-pseudo-orbit $\left(x_{i}\right)$ is periodic provided $x_{k n+r}=x_{r}$ for some $n \geq 1$ and all $k \geq 0,0 \leq r \leq n-1$. The period of $\left(x_{i}\right)$ is the smallest such $n$. The chainrecurrent set of $f$ is the set $R(f)$ consisting of points that are members of a periodic $\alpha$-pseudo-orbit for each $\alpha>0$.

Let $\mathscr{A}$ and $\mathscr{B}$ denote open covers of $X$ and let $N(\mathscr{A})$ denote the minimum cardinality of a subcover of $\mathscr{A}$. Define open covers by $f^{-k}(\mathscr{A})=$ $\left\{f^{-k}(U): U \in \mathscr{A}\right\}$ and $\mathscr{A} \vee \mathscr{B}=\{U \cap V: U \in \mathscr{A}, V \in \mathscr{B}\}$. Then the entropy of $f$ relative to the cover $\mathscr{A}$ is the number

$$
h(f, \mathscr{A})=\lim _{n \rightarrow \infty} \frac{1}{n} \log N\left(\mathscr{A} \vee f^{-1}(\mathscr{A}) \vee \cdots \vee f^{-(n-1)}(\mathscr{A})\right) .
$$

This limit, in fact, is the infimum. The topological entropy of $f$ is then

$$
h(f)=\sup \{h(f, \mathscr{A}): \mathscr{A} \text { is an open cover of } X\} .
$$

For compact metric spaces (as here)

$$
h(f)=\lim _{k \rightarrow \infty} h\left(f, \mathscr{A}_{\kappa}\right)
$$

for every collection $\left\{\mathscr{A}_{\kappa}\right\}$ of open covers with mesh going to zero as $k$ tends to infinity (cf. [DGS]).

The following definition of topological entropy, due to Bowen, is more readily adapted to pseudo-orbits. A subset $E$ of $X$ is $(n, \varepsilon)$-separated if, for each $x, y \in E, x \neq y$, there is a $k, 0 \leq k<n$, such that $d\left(f^{k}(x), f^{k}(y)\right)>\varepsilon$. Let $S(n, \varepsilon)$ denote the maximum cardinality of an $(n, \varepsilon)$-separated set in $X$ and define

$$
h(f, \varepsilon)=\limsup _{n \rightarrow \infty} \frac{1}{n} \log S(n, \varepsilon) .
$$

Then the topological entropy is given by [B2]

$$
h(f)=\lim _{\varepsilon \rightarrow \infty} h(f, \varepsilon) .
$$

We mimic this definition for pseudo-orbits by saying a collection $E$ of $\alpha$ pseudo-orbits is $(n, \varepsilon)$-separated if, for each $\left(x_{i}\right),\left(y_{i}\right) \in E,\left(x_{i}\right) \neq\left(y_{i}\right)$, there is a $k, 0 \leq k<n$, for which $d\left(x_{\kappa}, y_{\kappa}\right)>\varepsilon$. Let $S(n, \varepsilon, \alpha)$ denote the maximal cardinality of an $(n, \varepsilon)$-separated set of $\alpha$-pseudo-orbits. Note that 
$S(n, \varepsilon, \alpha)$ is finite by compactness of $X^{n}=X \underbrace{\times X \times \cdots \times X}_{n \text { times }}$. Now let

$$
\begin{gathered}
h_{\psi}(f, \varepsilon, \alpha)=\limsup _{n \rightarrow \infty} \frac{1}{n} \log S(n, \varepsilon, \alpha), \\
h_{\psi}(f, \varepsilon)=\lim _{\alpha \rightarrow 0} h_{\psi}(f, \varepsilon, \alpha),
\end{gathered}
$$

and

$$
h_{\psi}(f)=\lim _{\varepsilon \rightarrow 0} h_{\psi}(f, \varepsilon) .
$$

The number $h_{\psi}(f)$ will be called the pseudo-entropy of $f$-a temporary term, as we shall prove in the next section that $h_{\psi}(f)=h(f)$.

In many instances (Axiom A systems, for example) one can calculate topological entropy in terms of the growth rate of the number of periodic orbits of period $n$ as $n$ tends to infinity. We now formulate the growth rate of the number of periodic pseudo-orbits.

Let $E$ denote a set of $(n, \varepsilon)$-separated periodic $\alpha$-pseudo-orbits of period $n$. Thus, if $\left(x_{i}\right),\left(y_{i}\right) \in E,\left(x_{i}\right) \neq\left(y_{i}\right)$, then $\left(x_{i}\right)$ and $\left(y_{i}\right)$ are periodic of period $n$ and $d\left(x_{k}, y_{k}\right)>\varepsilon$ for some $k, 0 \leq k<n$. Let $P(n, \varepsilon, \alpha)$ denote the maximum cardinality of such a set. Now let

$$
\begin{gathered}
H_{\psi}(f, \varepsilon, \alpha)=\limsup _{n \rightarrow \infty} \frac{1}{n} \log P(n, \varepsilon, \alpha), \\
H_{\psi}(f, \varepsilon)=\lim _{\alpha \rightarrow 0} H_{\psi}(f, \varepsilon, \alpha),
\end{gathered}
$$

and

$$
H_{\psi}(f)=\lim _{\varepsilon \rightarrow 0} H_{\psi}(f, \varepsilon) .
$$

$H_{\psi}(f)$ is then the growth rate of the number of (separated) periodic $\alpha$-pseudoorbits of period $n$ as $n$ tends to infinity. We will show in the next section that $H_{\psi}(f)=h(f)$.

\section{PSEUDO-ORBITS AND ENTROPY}

Let $f: X \rightarrow X$ be a continuous map of the compact metric space $(X, d)$. In this section we prove the following results.

Theorem 1 (Misiurewicz). The topological entropy $h(f)$ is equal to the pseudoentropy $h_{\psi}(f)$.

Corollary (of Theorem 1). Let $f_{n}: X \rightarrow X$ be a sequence of continuous maps converging uniformly to $f$. Then

$$
\lim _{\varepsilon \rightarrow 0} \limsup _{n \rightarrow \infty} h\left(f_{n}, \varepsilon\right) \leq h(f) .
$$

Theorem 2. The topological entropy $h(f)$ is equal to the growth rate $H_{\psi}(f)$ of periodic pesudo-orbits.

The map $f: X \rightarrow X$ is said to have the shadowing property provided, for each $\alpha>0$, there is a $\beta>0$ such that if $\left(x_{i}\right)$ is an $\alpha$-pseudo-orbit, then there 
is an $x \in X$ with $d\left(f^{n}(x), x_{n}\right)<\beta$ for all $n \geq 0$. The homeomorphism $f$ is expansive if there is a constant $\varepsilon>0$ such that, for each $x, y \in X, x \neq$ $y, d\left(f^{n}(x), f^{n}(y)\right) \geq \varepsilon$ for some $n \in \mathbb{Z}$. Let $\operatorname{Fix}(g)$ denote the set of fixed points of a map $g$. The following well-known result (cf. [B3]) is an immediate corollary of Theorem 2 .

Corollary. If the expansive homeomorphism $f: X \rightarrow X$ has the shadowing property, then

$$
h(f)=\limsup _{n \rightarrow \infty} \frac{1}{n} \log \left[\# \operatorname{Fix}\left(f^{n}\right)\right] .
$$

Proof of Theorem 1. Given $\alpha \geq 0$, let

$$
Y_{\alpha}=\left\{\underline{x}=\left(\ldots x_{-1} ; x_{0}, x_{1}, \ldots\right) \mid d\left(f\left(x_{i-1}\right), x_{i}\right) \leq \alpha \text { for all } i \in \mathbb{Z}\right\}
$$

and define the metric $\underline{d}$ by

$$
\underline{d}(\underline{x}, \underline{y})=\sum_{i=-\infty}^{\infty} \frac{d\left(x_{i}, y_{i}\right)}{2^{|i|}}
$$

Note that each $Y_{\alpha}$ is a closed, and hence compact, subset of the product space $X^{\mathbb{Z}}$. Let $\sigma_{\alpha}: Y_{\alpha} \rightarrow Y_{\alpha}$ denote the shift $\sigma_{\alpha}\left(\cdots x_{-1} ; x_{0}, x_{1}, \ldots\right)=\left(\cdots x_{-1}, x_{0}\right.$; $\left.x_{1} \ldots\right)$. For each $\varepsilon>0$, let $\mathscr{A}_{1, \varepsilon}$ be a finite cover of $Y_{1}$ by $\varepsilon$-balls and form the restricted covers

$$
\mathscr{A}_{\alpha, \varepsilon}=\left\{A \cap Y_{\alpha} \mid A \in \mathscr{A}_{1, \varepsilon}\right\}, \quad 0 \leq \alpha \leq 1 .
$$

Lemma 1. $h\left(\sigma_{0}\right)=h(f)$.

Proof. Let $\pi_{k}: Y_{0} \rightarrow X$ be given by $\pi_{k}(\underline{x})=x_{k}, k \in \mathbb{Z}$. Since $f \circ \pi_{k}=\pi_{k} \circ \sigma_{0}$, it follows that $h(f) \leq h\left(\sigma_{0}\right)$. On the other hand, given a finite open cover $\mathscr{A}$ of $Y_{0}$, there is a $k \in \mathbb{Z}$ and a finite open cover $\mathscr{B}$ of $X$ such that $\pi_{k}^{-1}(\mathscr{B})$ refines $\mathscr{A}$. Such a $\mathscr{B}$ exists, since the collection $\left\{\pi_{j}^{-1}(U) \mid j \in \mathbb{Z}\right.$ and $U$ open in $X\}$ is a basis for the topology on $Y_{0}$. Then $h\left(\sigma_{0}, \mathscr{A}\right) \leq h\left(\sigma_{0}, \pi_{k}^{-1}(\mathscr{B})\right)=$ $h(f, \mathscr{B}) \leq h(f)$, so that $h\left(\sigma_{0}\right) \leq h(f)$.

Lemma 2. $h\left(\sigma_{0}, \mathscr{A}_{0, \varepsilon}\right) \geq \inf _{0<\alpha \leq 1} h\left(\sigma_{\alpha}, \mathscr{A}_{\alpha, \varepsilon}\right)$.

Proof. It follows from the definition of the entropy of a cover (cf. [DGS, 14.4]) that, for each $n>0$,

$$
h\left(\sigma_{\alpha}, \mathscr{A}_{\alpha, \varepsilon}\right) \leq \frac{1}{n} \log N\left(\bigvee_{i=0}^{n-1} \sigma_{\alpha}^{-i}\left(\mathscr{A}_{\alpha, \varepsilon}\right)\right) .
$$

Thus

since

$$
\inf _{0<\alpha \leq 1} h\left(\sigma_{\alpha}, \mathscr{A}_{\alpha, \varepsilon}\right) \leq \frac{1}{n} \log N\left(\bigvee_{i=0}^{n-1} \sigma_{0}^{-i}\left(\mathscr{A}_{0, \varepsilon}\right)\right)
$$

$$
N\left(\bigvee_{i=0}^{n-1} \sigma_{\alpha}^{-i}\left(\mathscr{A}_{\alpha, \varepsilon}\right)\right)
$$


decreases as $\alpha \rightarrow 0$ and equals

$$
N\left(\bigvee_{i=0}^{n-1} \sigma_{0}^{-i}\left(\mathscr{A}_{0, \varepsilon}\right)\right)
$$

for sufficiently small $\alpha>0$ ( $Y_{0}$ is compact). Letting $n \rightarrow \infty$, we have the desired inequality.

Lemma 3. $h_{\psi}(f, 2 \varepsilon, \alpha) \leq h\left(\sigma_{\alpha}, \mathscr{A}_{\alpha, \varepsilon}\right)$.

Proof. We can assume $f$ is surjective, for, otherwise, replace $X$ by $\bigcap_{n \geq 0} f^{n}(X)$ which carries the entropy of $f$. If $\left(x_{i}\right)$ and $\left(y_{i}\right)$ are $(n, 2 \varepsilon)$-separated $\alpha$ pseudo-orbits of $f$, then any two elements $\underline{x}$ and $\underline{y}$ of the form $\underline{x}=\left(\ldots ; x_{0}\right.$, $\left.x_{1}, \ldots\right)$ and $\underline{y}=\left(\ldots ; y_{0}, y_{1}, \ldots\right)$ are $(n, 2 \varepsilon)$-separated orbits of $\sigma_{\alpha}$. Let $\left\{B_{1}, B_{2}, \ldots, B_{k}\right\}$ be a minimal subcover of $\bigvee_{i=0}^{n-1} \sigma_{\alpha}^{-1}\left(\mathscr{A}_{\alpha, \varepsilon}\right)$. If there is a $j$ such that $\underline{x}, \underline{y} \in B_{j}$ then, for each $i=0,1, \ldots, n-1, \sigma_{\alpha}^{i}(\underline{x})$ and $\sigma_{\alpha}^{i}(\underline{y})$ lie in the same element of $\mathscr{A}_{\alpha, \varepsilon}$. That is, $\underline{d}\left(\sigma_{\alpha}^{i}(\underline{x}), \sigma_{\alpha}^{i}(\underline{y})\right)<2 \varepsilon$ for $i=$ $0,1, \ldots, n-1$. But $d\left(x_{i}, y_{i}\right) \leq \underline{d}\left(\sigma_{\alpha}^{i}(\underline{x}), \sigma_{\alpha}^{i}(\underline{y})\right)$ so we see that the maximum cardinality of an $(n, 2 \varepsilon)$-separated set of $\alpha$-pseudo-orbits for $f$ is not greater than $N\left(\mathrm{~V}_{i=0}^{n-1} \sigma_{\alpha}^{-i}\left(\mathscr{A}_{\alpha, \varepsilon}\right)\right)$. Thus $h_{\psi}(f, 2 \varepsilon, \alpha) \leq h\left(\sigma_{\alpha}, \mathscr{A}_{\alpha, \varepsilon}\right)$.

Now, for the proof of Theorem 1 , the inequality $h(f) \leq h_{\psi}(f)$ is apparent. For the reverse inequality,

$$
h_{\psi}(f, 2 \varepsilon, \alpha) \leq h\left(\sigma_{\alpha}, \mathscr{A}_{\alpha, \varepsilon}\right) \quad \text { Lemma } 3
$$

so that

$$
\begin{aligned}
h_{\psi}(f, 2 \varepsilon) & \leq \inf _{0<\alpha \leq 1} h\left(\sigma_{\alpha}, \mathscr{A}_{\alpha, \varepsilon}\right) \\
& \leq h\left(\sigma_{0}, \mathscr{A}_{0, \varepsilon}\right) \quad \text { Lemma } 2 .
\end{aligned}
$$

Letting $\varepsilon \rightarrow 0$ and using Lemma 1 , we get $h_{\psi}(f) \leq h(f)$.

Proof of the Corollary to Theorem 1. A true orbit of a map $g$ near $f$ is a pseudo-orbit of $f$. Thus, for each $\alpha<0$, there is an $N$ such that $n \geq N$ implies $h\left(f_{n}, \varepsilon\right) \leq h_{\psi}(f, \varepsilon, \alpha)$ for each $\varepsilon>0$. It follows that

$$
\limsup _{n \rightarrow \infty} h\left(f_{n}, \varepsilon\right) \leq h_{\psi}(f, \varepsilon) \text {. }
$$

Not let $\varepsilon \rightarrow 0$ for the desired inequality.

Proof of Theorem 2. The chain-recurrent set $R=R(f)$ is invariant under $f$ and contains the nonwandering set $\Omega$. Since $h(f)=h\left(\left.f\right|_{\Omega}\right)$ [B3] it follows that $h(f)=h\left(\left.f\right|_{R}\right)$.

The following lemma appears in [Ro].

Lemma 4. $R\left(\left.f\right|_{R}\right)=R(f)$.

Proof. Let $x \in R(f)$ and, for each $n \geq 1$, let $C_{n}=\left(x_{0}^{n}, \ldots, x_{m(n)}^{n}\right)$ be a $1 / n$-chain with $x_{0}^{n}=x=x_{m}^{n}$. Choose a subsequence $C_{n_{i}}$ that converges to 
a set $C$ in the Hausdorff metric $\delta$. It can be easily checked that $C \subset R(f)$. Now let $\alpha>0$ be given and let $\varepsilon>0$ be small enough so that $\varepsilon<\alpha / 3$ and if $d(y, z)<\varepsilon$ then $d(f(y), f(z))<\alpha / 3$. Let $i$ be large enough so that $1 / n_{i}<\alpha / 3$ and $\delta\left(C_{n_{i}}, C\right)<\varepsilon$. For each $k=0,1, \ldots, m\left(n_{i}\right)=m$, let $\tilde{x}_{i} \in C$ satisfy $d\left(\tilde{x}_{k}, x_{k}^{n_{i}}\right)<\varepsilon$. Then

$$
\begin{aligned}
d\left(f\left(\tilde{x}_{k}\right), \tilde{x}_{k+1}\right) \leq & d\left(f\left(\tilde{x}_{k}\right), f\left(x_{k}^{n_{i}}\right)\right)+d\left(f\left(x_{k}^{n_{i}}, x_{k+1}^{n_{i}}\right)\right. \\
& +d\left(x_{k+1}^{n_{i}}, \tilde{x}_{k+1}\right) \\
< & \alpha / 3+\alpha / 3+\alpha / 3=\alpha .
\end{aligned}
$$

Choose $\tilde{x}_{0}=x=\tilde{x}_{m}$. Then $\left(\tilde{x}_{0}, \tilde{x}_{1}, \ldots, \tilde{x}_{m}\right)$ is an $\alpha$-chain in $C \subset R(f)$ from $x$ to $x$.

Two points $x, y \in R=R(f)$ are said to be in the same $\alpha$-chain-transitive component of $R$ provided there is an $\alpha$-chain (in $R$ ) from $x$ to $y$ and an $\alpha$-chain (in $R$ ) from $y$ to $x$. If $\varepsilon>0$ is such that $d(x, y)<\varepsilon$ implies $d(f(x), f(y))<\alpha$, then, for $x, y \in R, d(x, y)<\varepsilon$ implies that $x$ and $y$ are in the same $\alpha$-chain-transitive component of $R$. It follows that the $\alpha$ chain-transitive components of $R$ are simultaneously open and closed in $R$, partition $R$, are finite in number, and each is invariant under $f$. Thus $h(f)=$ $h\left(\left.f\right|_{R}\right)=h\left(\left.f\right|_{T}\right)$ for some $\alpha$-chain-transitive component $T$ of $R$.

Lemma 5. If $T$ is an $\alpha$-chain-transitive component of $R$, there is a positive integer $m=m(T)$ such that for every pair $x, y$ in $T$ there is a $2 \alpha$-chain in $T$ from $x$ to $y$ of length less than or equal to $m$.

Proof. Given $(x, y) \in T \times T$ there is a $k=k(x, y)$ such that there is an $\alpha$-chain of length $k$ in $T$ from $x$ to $y$. There are then neighborhoods $U_{x}$ of $x$ in $T$ and $U_{y}$ of $y$ in $T$ such that if $x^{\prime} \in U_{x}$ and $y^{\prime} \in U_{y}$ then there is a $2 \alpha$-chain in $T$ of length $k$ from $x^{\prime}$ to $y^{\prime}$. Cover the compact $T \times T$ by a finite collection of sets $U_{x_{i}} \times U_{y_{i}}$ and let $m=\max \left\{k\left(x_{i}, y_{i}\right)\right\}$.

Given an $\alpha$-chain-transitive component $T$ of $R=R(f)$, let $\operatorname{Per}_{T}(n, \varepsilon, \beta)$ denote the maximal size of an $(n, \varepsilon)$-separated set of periodic $\beta$-pseudo-orbits in $T$ of period $n$ and let $S_{T}(n, \varepsilon, \beta)$ denote the maximum cardinality of an $(n, \varepsilon)$-separated set of $\beta$-pseudo-orbits in $T$. The following is an immediate consequence of Lemma 5.

Lemma 6. Let $m=m(T)$ be as in Lemma 5. Then

$$
S_{T}(n, \varepsilon, \alpha) \leq \sum_{i=1}^{m+n} \operatorname{Per}_{T}(i, \varepsilon, 2 \alpha) .
$$

Now, for the proof of Theorem 2, let $T$ be an $\alpha$-chain-transitive component of $R=R(f)$ such that $h(f)=h\left(\left.f\right|_{T}\right)$ and let $m=m(T)$ be as in Lemma 5 . For $k \geq 1$, let $i_{k}, 1 \leq i_{k} \leq k$, be such that $\operatorname{Per}_{T}\left(i_{k}, \varepsilon, 2 \alpha\right) \geq \operatorname{Per}_{T}(i, \varepsilon, 2 \alpha)$ 
for all $1 \leq i \leq k$. We have

$$
\begin{aligned}
h(f)=h\left(\left.f\right|_{T}\right) & =h_{\psi}\left(\left.f\right|_{T}\right) \quad(\text { Theorem 1) } \\
& \leq \limsup _{n \rightarrow \infty} \frac{1}{n} \log S_{T}(n, \varepsilon, \alpha) \\
& \leq \limsup _{n \rightarrow \infty} \frac{1}{n} \log \sum_{i=1}^{m+n} \operatorname{Per}_{T}(i, \varepsilon, 2 \alpha) \quad(\text { Lemma 6) } \\
& \leq \limsup _{n \rightarrow \infty} \frac{1}{m+n} \log \left[(m+n) \operatorname{Per}_{T}\left(i_{m+n}, \varepsilon, 2 \alpha\right)\right] \\
& \leq \limsup _{n \rightarrow \infty} \frac{1}{i_{m+n}} \log \operatorname{Per}_{T}\left(i_{m+n}, \varepsilon, 2 \alpha\right) \\
& \leq H_{\psi}(f, \varepsilon, 2 \alpha) .
\end{aligned}
$$

Letting $\alpha$, then $\varepsilon$, go to zero we have $h(f) \leq H_{\psi}(f)$. That $H_{\psi}(f) \leq h_{\psi}(f)$ is clear from definitions so that $H_{\psi}(f) \leq h(f)$ by Theorem 1 .

\section{REFERENCES}

[AKM] R. L. Adler, A. G. Konheim, and M. H. McAndrew, Topological entropy, Trans. Amer. Math. Soc. 114 (1965), 309-319.

[B1] R. Bowen, Equilibrium states and the ergodic theory of Axiom A diffeomorphisms, Lecture Notes in Math., vol. 470, Springer-Verlag, New York, 1975.

[B2] _ Entropy for group endomorphisms and homogeneous spaces, Trans. Amer. Math. Soc. 153 (1971), 401-414; 181 (1973), 509-510.

[B3] _ Topological entropy and Axiom A, Proc. Sympos. Pure Math., vol. 14, American Mathematical Society, Providence, RI, 1970, pp. 23-42.

[BS] M. Barge and R. Swanson, Rotation shadowing properties of circle and annulus maps, Ergodic Theory Dynamical Systems 8 (1988), 509-521.

[C] C. Conley, Isolated invariant sets and the Morse Index, CBMS Conference Series No. 38 (1978).

[DGS] M. Denker, C. Grillenberger, and K. Sigmund, Ergodic theory on compact spaces, Lecture Notes in Math., vol. 527, Springer-Verlag, New York, 1976.

[E] R. Easton, Isolating blocks and epsilon chains for maps, preprint.

[F] J. Franks, Realizing rotation vectors for torus homeomorphisms, Trans. Amer. Math. Soc. 311 (1989), 107-115.

[HYG1] S. M. Hammel, J. A. Yorke, and C. Grebogi, Do numerical orbits of chaotic dynamical processes represent true orbits? J. Complexity 3 (1987), 136-145.

[HYG2] _ Numerical orbits of chaotic processes represent true orbits, Bull. Amer. Math. Soc. 19 (1988), 465-469.

[H] M. Hurley, Attractors in cellular automata, Ergodic Theory Dynamical Systems (to appear).

[Mc] R. McGehee, Some metric properties of attractors with applications to computer simulations of dynamical systems, preprint. 
[Mi] M. Misiurewicz, Remark on the definition of topological entropy, Dynamical Systems and Partial Differential Equations (Caracas, 1984), Caracas, 1986, 65-67.

[R] M. Rees, A minimal positive entropy homeomorphism of the 2-torus, J. London Math. Soc. (2) 23 (1981), 537-550.

[Ro] C. Robinson, Stability theorems and hyperbolicity in dynamical systems, Rocky Mountain J. Math. 7 (1977), 425-437.

Department of Mathematical Sciences, Montana State University, Bozeman, MonTANA 59717 